\title{
Aprendizado Prático de Subsistemas de Entrada/Saída em Projetos de Sistemas Computacionais com Suporte do Simulador CompSim
}

\author{
Guilherme Álvaro Esmeraldo¹, Edson Lisboa², Lucas Cartaxo¹, Cícero Samuel Mendes \\ ${ }^{1}$ Laboratório de Sistemas Embarcados e Distribuídos - LEDS/IFCE \\ Instituto Federal do Ceará - Campus Crato \\ ${ }^{2}$ Laboratório de Estudos Avançados em Eletrônica - LEA/IFS \\ Instituto Federal de Sergipe - Campus Aracaju \\ guilhermealvaro@ifce.edu.br, edson.lisboa@academico.ifs.edu.br, \{lfonteesc,mr.samuelmendes\}@gmail.com
}

\begin{abstract}
Resumo: No estudo de projetos de sistemas computacionais, o tema Subsistemas de Entrada/Saída inclui conceitos complexos e extensos, que não são tratados de forma adequada pelos simuladores computacionais presentes na literatura. Este artigo apresenta uma proposta de uso do simulador computacional CompSim para suportar os processos de ensino-aprendizagem práticos em Subsistemas de Entrada/Saída. Para o desenvolvimento do trabalho aqui proposto, realizou-se um levantamento bibliográfico de aspectos de subsistemas de entrada/saída, e de como os simuladores e sistemas computacionais reais os tratam. Com isso, formulouse mecanismos que simplificam a criação e conexão de novos periféricos ao sistema computacional virtual do CompSim. A abordagem proposta tem sido empregada em diferentes cursos e os resultados mostram a efetividade do processo de ensinoaprendizagem.
\end{abstract}

Palavras-chave: Subsistema de Entrada/Saída; Aprendizado Prático; Simulador CompSim.

\section{Introdução}

Em cursos técnicos e superiores nas áreas de computação e engenharia eletrônica, há disciplinas que tratam de aspectos de projetos de sistemas computacionais, tal como a de Arquitetura e Organização de Computadores (AOC)[1][2]. Nessas disciplinas, geralmente, os conteúdos trabalhados incluem desde as estruturas e comportamento dos componentes do computador até sua programação em linguagem de baixo nível.

Nesses conteúdos, um dos aspectos mais importantes se refere à interação do sistema computacional com seus periféricos. Essa interação envolve diversos elementos como interfaces, barramentos, modelos e protocolos de comunicação, módulos de entrada/saída e os próprios periféricos. Esses conceitos são considerados complexos e não triviais, bem como têm sido frequentemente abordados de forma puramente conceitual e com uso de abstrações [3].

$\mathrm{Na}$ literatura tem-se optado pela utilização de simuladores para apoio ao aprendizado dos diferentes aspectos de projetos de sistemas computacionais [4]. Os simuladores são ferramentas que buscam representar cenários reais no projeto de sistemas computacionais e têm como principais benefícios a abstração dos diferentes recursos do computador, não necessitarem de laboratórios de hardware e técnicos especializados, bem como permitem configuração e feedback rápidos nas simulações.

Vários estudos, como os apresentados em Pena e Freitas [5] e Esmeraldo et al. [7], realizaram comparativos entre simuladores da literatura, onde consideraram determinadas métricas comparativas, como, por exemplo, alto desempenho, suporte de interface gráfica, disponibilidade de documentação, distribuição livre, entre outras. Observa-se todavia que os simuladores: ou não apresentam todos os recursos didáticos necessários para apoio à disciplina [5]; ou, devido à adoção de abstrações, não abordam adequadamente conteúdos importantes [3]; ou, por focarem em determinados componentes do computador, há a necessidade de se utilizar mais de um simulador [4]; ou buscam fidelizar as características dos componentes do computador, tornando-se complexos de configurar e interagir [6].

Nesse sentido, este artigo apresenta um novo recurso do simulador CompSim [11], o qual vem com a proposta de apoiar o aprendizado e exploração, de forma prática, da interação entre o computador e os periféricos. Esse recurso consiste de uma interface de Entrada/Saída (E/S) padronizada que permite conectar, de forma simplificada e automatizada, o sistema computacional simulado à diferentes periféricos, com objetivo de apoiar a realização de experimentos que envolvam desde o projeto de periféricos virtuais e físicos até sistemas computacionais completos reais.

\section{Fundamentação Teórica}

Dispositivos de E/S são fundamentais em sistemas computacionais, pois possibilitam a interface para a interação entre o sistema e o ambiente exterior. Tais interações podem se configurar entre homem-máquina (IHM), máquina-máquina (IMM) e de comunicação [8]. Portanto, dispositivos periféricos apresentam grandes variações com relação às funcionalidades, tecnologias empregadas, diferentes taxas de transmissão de dados, além de tamanho e formato dos dados na comunicação. Essa heterogeneidade inviabiliza que o processador trate diretamente as particularidades de cada dispositivo periférico. Assim, o projeto de subsistemas de E/S é uma solução integrada de hardware e software para cada dispositivo específico a ser conectado ao sistema.

De uma forma geral, um Subsistema de E/S é composto por dois submódulos: Módulo de E/S, também conhecido como Controlador, e o próprio periférico, também conhecido como dispositivo externo. A Figura 1 ilustra esses dois elementos e como estão relacionados. 


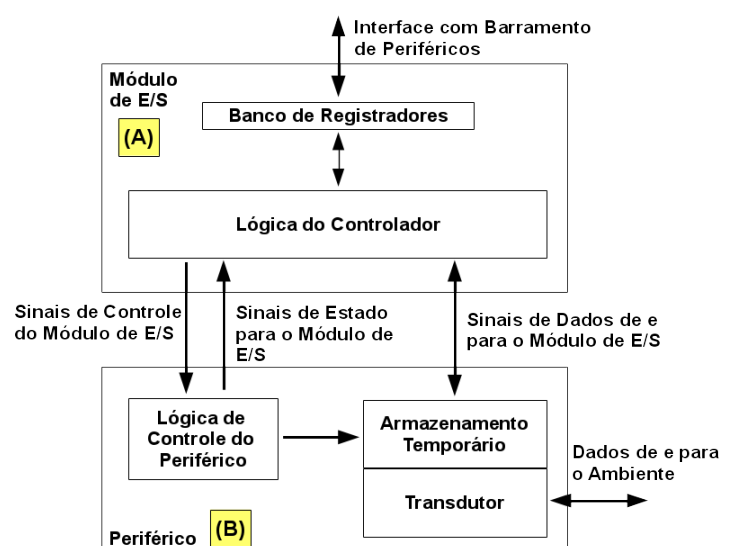

Figura 1. Subsistema de E/S.

O Módulo de E/S, que faz interface com o barramento de periféricos, possui um Banco de Registradores - que armazenam dados, informações de controle, comandos e estados - e a Lógica do Controlador das funções do periférico, como pode ser visto na Figura 1(A). Já o periférico, ilustrado na Figura 1(B) contém: 1) Lógica de Controle do Periférico, que se encarrega de interpretar comandos enviados pelo Módulo de E/S e faz com que a função relativa ao comando seja executada; 2) Transdutor, que é necessário para fazer as devidas conversões entre sinais de natureza diferentes (e.g. conversão entre sinal elétrico proveniente do sistema computacional em uma forma de energia compatível com o ambiente externo, tais como ondas magnéticas ou sonoras); e 3) Armazenamento temporário, que está associado ao Transdutor para que os dados possam ser transferidos entre o periférico e o Módulo de E/S.

Evidentemente, que os Subsistemas de E/S variam com a natureza e complexidade do periférico. Desta forma, percebe-se que o estudo, compreensão e desenvolvimento não são atividades imediatas. Assim, plataformas virtuais têm surgido como um mecanismo para otimizar tanto os processos de ensinoaprendizagem quanto para aumentar o desempenho no desenvolvimento de software e hardware, em projetos de sistemas computacionais, tal como será apresentado a seguir.

\section{O Simulador CompSim}

CompSim é um simulador de sistema completo - são tipos de simuladores que incluem todos os componentes do computador -, o qual inclui uma plataforma de hardware simulável, conhecida por "Mandacaru”, a qual inclui os seguintes componentes computacionais de simulação: 1) CPU: um processador de 16-bits, com arquitetura mista (RISC e CISC), composta por 16 instruções para realização de operações de transferência de dados, lógicas e aritméticas, desvio de fluxo de programa e de entrada/saída com periféricos. Possui ainda os seguintes submódulos: banco de registradores, contador de instrução, unidade de controle, unidade lógica e aritmética e subsistema de tratamento de interrupções de software e hardware; 2) Memória cache: é utilizada para otimizar o desempenho dos programas (aproveitando as características de localidade espacial e temporal) e suporta diferentes tipos de configuração, como de políticas de mapeamento, de substituição, entre outras; 3) Memória RAM: é utilizada para armazenamento de dados, instruções e pilha dos programas que serão executados no simulador; 4) Barramentos: o simulador inclui dois barramentos, sendo um de Sistema, que permite a comunicação entre o processador e as memórias Cache e RAM, e um de Periféricos, que permite que o processador se comunique com o Subsistema de Entrada/Saída; 5) Subsistema de Entrada/Saída: inclui uma interface padronizada com módulos de entrada/saída, que possibilita a comunicação do processador com diferentes tipos de periféricos.

O simulador também conta com uma interface gráfica para configurar os componentes virtuais da plataforma Mandacaru, ajustar parâmetros de simulação e suportar a codificação de aplicações em baixo nível (Assembly).

A Figura 2 mostra a interface gráfica do CompSim, na qual pode-se visualizar os seguintes componentes gráficos: A) Editor de código; B) Processador; C) Memória cache; D) Memória RAM; e E) Componentes de controle de configuração e execução de simulação.

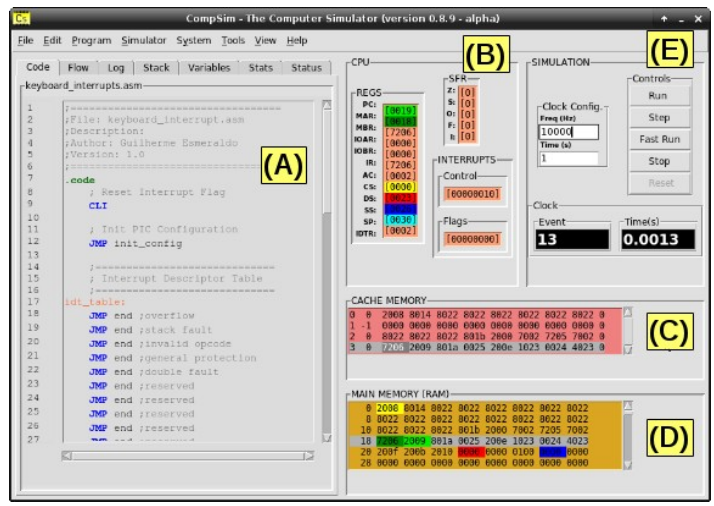

Figura 2. Interface Gráfica do CompSim.

\subsection{Subsistema de Entrada/Saída}

O Subsistema de E/S, presente na plataforma de hardware virtual do CompSim, permite que novos periféricos sejam conectados automaticamente ao barramento de periféricos. Para tanto, cada periférico deve incluir dois arquivos: 1) o primeiro (arquivo com extensão “.csd”) contém uma especificação de sua interface, a qual inclui as seguintes definições: número(s) da(s) porta(s) de entrada/saída; número da interrupção (IRQ), caso o periférico suporte; dados para instanciar o componente de software (nome do pacote e da classe de software); e uma curta descrição textual do respectivo periférico; 2) o segundo arquivo inclui o programa que será utilizado para emular o comportamento do periférico (o código-fonte do programa, com extensão “.py”, descrito na linguagem Python).

Desta forma, ao se criar uma plataforma computacional no simulador CompSim, seu Subsistema de E/S buscará pelos arquivos de descrição de interface de componente (“.csd”) e, de forma automatizada, 
instanciará os respectivos componentes de software (“.py”), que serão conectados ao barramento virtual de periféricos (esse processo é ilustrado na Figura 3). Com isso, os novos periféricos já estarão prontos para interação com restante dos componentes de hardware do sistema computacional simulado.

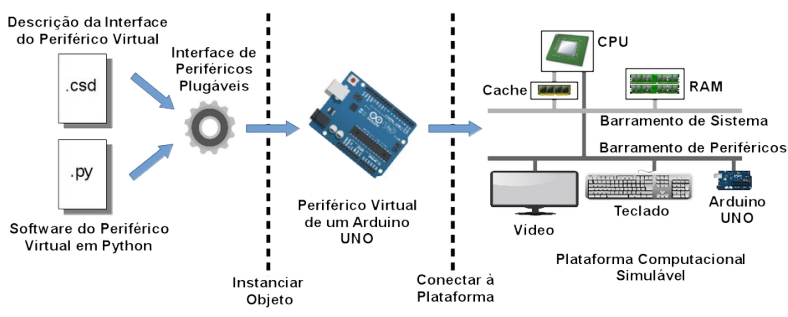

Figura 3. Criação de um periférico plugável e conexão à plataforma virtual.

O CompSim inclui um assistente para dar suporte à criação de novos periféricos, chamado de "Device Interface Creator” (ilustrado na Figura 4). Basicamente, ele consiste de um formulário, que deve ser preenchido, pelo projetista, com as informações da interface do novo periférico. Em seguida, o assistente gera automaticamente os respectivos arquivos “.csd” e “.py”, ou, em outras palavras, cria o respectivo Módulo de E/S. Deste ponto em diante, para concluir a criação do novo periférico, o projetista deve apenas focar no desenvolvimento de seu comportamento funcional, ao complementar sua codificação no arquivo-fonte “.py”.

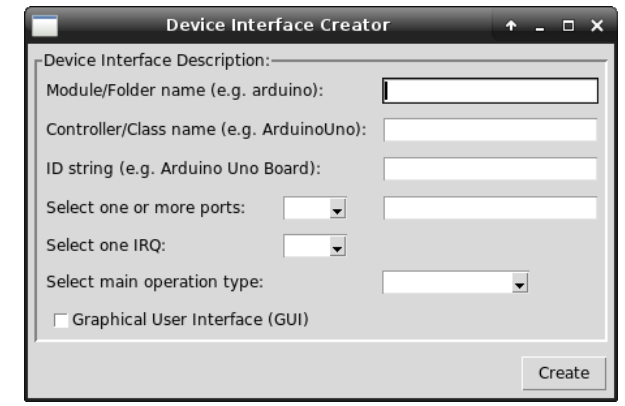

Figura 4. Assistente de criação de interface de periféricos.

Atualmente, o CompSim conta oficialmente com os periféricos Teclado, Vídeo e Arduíno UNO, como podem ser vistos nas Figuras 5(A), (B) e (C), respectivamente. Os periféricos Teclado e Vídeo foram definidos como virtuais (desenvolvidos apenas em software), pois emulam os comportamentos dos reais. Cabe ressaltar, que o periférico Teclado possui duas versões, onde diferem pelo mecanismo de interação com o processador (um por polling e o outro por interrupções). Já o periférico Arduíno UNO é dividido em dois submódulos, sendo que: o primeiro (software) implementa, em software, o Módulo de E/S e permite integração do dispositivo ao barramento de periféricos; e o segundo submódulo, que é o periférico propriamente dito, consiste de uma board física da plataforma aberta de prototipação Arduíno modelo UNO [9]. Desta forma, é possível criar cenários de projetos de sistemas computacionais onde pode-se realizar a simulação da plataforma computacional virtual interagindo com hardware real. Com o apoio do Arduíno UNO, é possível criar, de forma simplificada, diferentes periféricos físicos para a plataforma virtual, com apoio de diferentes componentes eletrônicos, tais como resistores, capacitores, chaves, leds, displays, sensores, motores, entre outros.

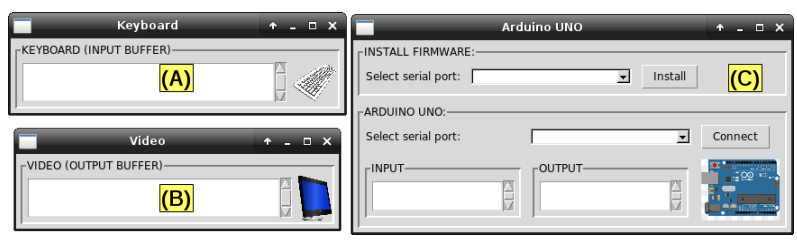

Figura 5. Periféricos do CompSim.

\section{Metodologia}

Após um levantamento do estado da arte em simuladores computacionais, verificou-se que o tema Subsistemas de E/S ou não é tratado adequadamente na maioria dos simuladores utiliza-se abstrações que, em certa medida, tratam os conceitos fundamentais tangencialmente - ou não é abordado.

Nesse sentido, para o desenvolvimento do Subsistema de E/S do simulador CompSim, inicialmente, foi necessário realizar um levantamento teórico aprofundado sobre o tema de E/S em projetos de sistemas computacionais. Nesse estudo, avaliou-se a arquitetura de diferentes processadores, multiprocessadores e microcontroladores reais, onde levantou-se suas principais características relacionadas à E/S. Em seguida, buscou-se aplicar abordagens de modularização (desenvolvimento baseado em componentes, padrões de projeto, entre outros) para definir a interface dos componentes de software que modelam os periféricos. Com isso, foi possível simplificar o desenvolvimento de novos periféricos e torná-los automaticamente conectáveis ao barramento virtual de periféricos do CompSim.

Para avaliação dos mecanismos definidos para o Subsistema de E/S proposto, foram desenvolvidos exemplos de periféricos (ver seção anterior), os quais são utilizados como base na criação de novos. Uma vez que se pode disponibilizar, aos estudantes, os códigosfonte dos periféricos apresentados na seção anterior, é possível tomá-los como base e utilizar seus principais conceitos de interface na criação de novos periféricos.

Por fim, a avaliação do suporte ao processo de ensino-aprendizagem é realizada através da aplicação de uma rubrica [10], onde os estudantes avaliam o suporte educacional e a experiência de uso do simulador. Da mesma forma, os professores das disciplinas avaliam se o suporte do simulador favorece diferentes aspectos pedagógicos.

\section{Discussão}

O Simulador CompSim tem sido utilizado por estudantes de diferentes turmas de um curso de Técnico em Eletrônica e de um bacharelado em Sistemas de Informação. Com o Subsistema de E/S do CompSim, os estudantes lidam de maneira prática com os conceitos de 
E/S na criação de diferentes tipos de periféricos virtuais (software) e físicos (software e hardware), bem como nos aspectos de sua programação, através da criação dos respectivos device drivers.

Entre os periféricos criados pelos estudantes, é possível agrupá-los em três categorias básicas, com respectivos exemplos: 1) Periféricos virtuais: sintetizadores de sons com uso do buzzer do computador, emuladores de periféricos, tais como display de 7 segmentos, display LCD 16x2, teclados numéricos, chaves e botões, e um Arduíno UNO virtual; 2) Periféricos físicos analógicos: com o suporte da board do Arduíno UNO e componentes eletrônicos, foi possível criar periféricos físicos para interação do usuário com os programas que executam no simulador, tais como saída digital em leds e displays de 7 segmentos, entrada de dados digitais pelo uso de teclados numéricos e chaves tácteis, e entrada analógica pelo uso de potenciômetros e sensores (luz, temperatura e umidade); 3) Periféricos físicos digitais: com suporte de uma board do Arduíno UNO e uma board com FPGA (lógica digital reconfigurável), foi possível criar dispositivos digitais, tais como contadores digitais e registradores (buffer, deslocamento e carregamento paralelo de dados).

Ao final das disciplinas, um total de 54 estudantes se voluntariou para avaliar o processo de aprendizado de Subsistemas de E/S pelo uso dos recursos do CompSim. Numa escala de 1 a 4 , eles destacaram positivamente que: 1) favoreceu o aprendizado de conceitos em E/S pelo desenvolvimento do pensamento de alto nível (méd. 3,8, desv. pad. 0,5); 2) dinamizou o ambiente de laboratório (méd. 3,8, desv. pad. 0,4); 3) adequou-se aos conteúdos curriculares dos respectivos cursos e ao nível escolar (méd. 3,7, desv. pad. 0,5); e 4) a disponibilidade de materiais complementares, tais como componentes eletrônicos e exemplos de códigos-fonte, permitiu auxiliar o aprendizado no uso do simulador e dos conteúdos curriculares (méd. 3,7, desv. pad. 0,4). Do ponto de vista pedagógico, foi possível constatar um aumento significativo na motivação, participação em aulas práticas, produtividade e desempenho geral das turmas, além da redução da evasão nas disciplinas.

\section{Conclusões}

Este artigo apresenta uma proposta de uso do simulador CompSim para suporte ao aprendizado de conceitos de Subsistemas de E/S em projetos de sistemas computacionais. O simulador proposto inclui uma plataforma virtual de hardware e uma interface de software de E/S que permite conectar novos periféricos (virtuais e físicos), de forma automatizada, à plataforma.

Durante as práticas laboratoriais, os estudantes são estimulados a criar periféricos diversificados, utilizando diferentes recursos (e.g. linguagem de programação Python, Arduíno UNO e componentes eletrônicos), e com isso aplicar os conceitos aprendidos nas aulas teóricas. Os resultados mostraram que a abordagem proposta trouxe resultados efetivos no processo de ensino-aprendizagem em Subsistemas de E/S, além de aumentar a motivação e participação, e reduzir a evasão.

\section{Bibliografia}

[1] ACM. Association for Computing Machinery; IEEE Computer Society. (2013) Curriculum Guidelines for Undergraduate Degree Programs in Computer Science.

[2] Zorzo, A. F.; Nunes, D.; Matos, E.; Steinmacher, I.; Leite, J.; Araujo, R. M.; Correia, R.; Martins, S. (2017) Referenciais de formação para os cursos de graduação em computação. In: Sociedade Brasileira de Computação (SBC), 153p.

[3] Larraza-Mendiluze, E.; Garay-Vitoria, N. (2014) Approaches and tools used to teach the computer input/output subsystem: A survey. IEEE Transactions on Education 58(1): 1-6. DOI: 10.1109/TE.2014.2310711.

[4] Fernandes, S. R.; Silva, I. S. (2017) Relato de Experiência Interdisciplinar Usando MIPS. International Journal of Computer Architecture Education (IJCAE) 6(1): 52-61.

[5] Penna, P. H; Freitas, H. C. (2013) Análise e Avaliação de Simuladores de Sistemas Completos para o Ensino de Arquitetura de Computadores. International Journal of Computer Architecture Education (IJCAE) 2(1): 13-16.

[6] Duenha, L.; Azevedo, R. (2016) Utilização dos Simuladores do MPSoCBench para o Ensino e Aprendizagem de Arquitetura de Computadores. In: International Journal of Computer Architecture Education (IJCAE) 5(1): 26-31.

[7] Esmeraldo, G. A. R. M.; Mendes, C. S. R. ; Cartaxo, L. F.; Lisboa, E. B. (2019) Apoio ao Aprendizado em Arquitetura e Organização de Computadores: Um Estudo Comparativo entre Simuladores Computacionais. Revista Tecnologias na Educação 31: 1-17.

[8] Stallings, W. (2017) Computer Organization and Architecture. 10th Edition. Pearson.

[9] Badamasi, Y. A. (2014). The working principle of an Arduino. In: Proc. 2014 11th International Conference on Electronics, Computer and Computation (ICECCO), p. 14. IEEE.

[10] Yuan, M.; Recker, M. (2015) Not all rubrics are equal: A review of rubrics for evaluating the quality of open educational resources. The International Review of Research in Open and Distributed Learning 16(5): 16-38.

[11] COMPSIM. CompSim - The Computer Simulator. Disponível em: <http://compsim.crato.ifce.edu.br/>. Acesso em: 20 maio 2020. 